\title{
A Predictive Model for Nonsentinel Node Status after Sentinel Lymph Node Biopsy in Sentinel Lymph Node-Positive Chinese Women with Early Breast Cancer
}

\author{
Lifang He $\mathbb{D}^{1},{ }^{1,2}$ Peide Liang $\mathbb{D}^{1,3}$ Huancheng Zeng ${ }^{10},{ }^{1}$ Guangsheng Huang $\mathbb{D}^{1}$,

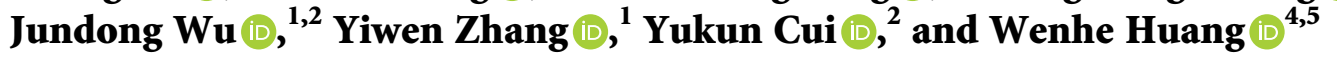 \\ ${ }^{1}$ Breast Center, Cancer Hospital of Shantou University Medical College, Shantou 515000, Guangdong Province, China \\ ${ }^{2}$ Guangdong Provincial Key Laboratory for Breast Cancer Diagnosis and Treatment, \\ Cancer Hospital of Shantou University Medical College, Shantou 515000, Guangdong Province, China \\ ${ }^{3}$ Department of Thyroid and Breast Surgery, Dongguan Houjie Hospital, Dongguan 523000, Guangdong Province, China \\ ${ }^{4}$ Department of Breast and Thyroid Surgery, Xiang'an Hospital of Xiamen University, No. 2000, Xiang'an East Road, \\ Xiamen 361101, Fujian Province, China \\ ${ }^{5}$ Key Laboratory for Endocrine-Related Cancer Precision Medicine of Xiamen, Xiamen 361101, Fujian Province, China
}

Correspondence should be addressed to Yukun Cui; 2461792142@qq.com and Wenhe Huang; huangwenhe2009@163.com

Received 16 December 2021; Accepted 11 January 2022; Published 24 February 2022

Academic Editor: Dong-Hua Yang

Copyright $(\odot 2022$ Lifang He et al. This is an open access article distributed under the Creative Commons Attribution License, which permits unrestricted use, distribution, and reproduction in any medium, provided the original work is properly cited.

Background. Axial lymph node dissection (ALND) is needed in patients with positive sentinel lymph node (SLN). ALND is easy to cause upper limb edema. Therefore, accurate prediction of nonsentinel lymph nodes (non-SLN) which may not need ALND can avoid excessive dissection and reduce complications. We constructed a new prognostic model to predict the non-SLN metastasis of Chinese breast cancer patients. Methods. We enrolled 736 patients who underwent sentinel lymph node biopsy (SLNB); 228 (30.98\%) were diagnosed with SLNB metastasis which was determined by intraoperative pathological detection and further accepted ALND. We constructed a prediction model by univariate analysis, multivariate analysis, " $R$ " language, and binary logistic regression in the abovementioned 228 patients and verified this prediction model in 60 patients. Results. Based on univariate analysis using $\alpha=0.05$ as the significance level for type I error, we found that age $(P=0.045)$, tumor size $(P=0.006)$, multifocality $(P=0.011)$, lymphovascular invasion $(P=0.003)$, positive SLN number $(P=0.009)$, and negative SLN number $(P=0.034)$ were statistically significant. Age was excluded in multivariate analysis, and we constructed a predictive equation to assess the risk of non-SLN metastasis: $\operatorname{Logit}(P)=\operatorname{Ln}(P / 1-P)=0.267 * a+1.443 * b+1.078 * c+0.471 * d-0.618 * e-2.541$ (where " $a$ " represents tumor size, " $b$ " represents multifocality, " $c$ " represents lymphovascular invasion, " $d$ " represents the number of metastasis of SLN, and " $e$ " represents the number of SLNs without metastasis). AUCs for the training group and validation group were 0.715 and 0.744 , respectively. When setting the risk value below $22.3 \%$, as per the prediction equation's low-risk interval, our model predicted that about $4 \%$ of patients could avoid ALND. Conclusions. This study established a model which demonstrated good prognostic performance in assessing the risk of non-SLN metastasis in Chinese patients with positive SLNs.

\section{Introduction}

In the 2021 report, the International Agency for Research on Cancer (IARC) showed there were 2.26 million new breast cancer cases in 2020 worldwide, comprising the world's largest cancer incidence, with a mortality rate that remains the second leading cause of death for women with cancer.
China's annual incidence of new breast cancer cases is 420,000 , accounting for $18.6 \%$ of the world's total new breast cancer cases, and it is also the largest number of new cancer cases for women in China [1].

Early-stage breast cancer is mainly treated with surgery, chemotherapy, endocrine therapy, radiotherapy, or targeted therapy $[2,3]$. Axillary lymph node dissection (ALND) has 
been a standard method in breast cancer surgery, since the inception of Halsted radical mastectomy, and has always been the gold standard for assessing axillary lymph node metastasis and determining cancer stage. However, its complications, such as upper limb dysfunction, upper arm lymphedema, and sensory disorders, seriously reduce the patients' quality of life [4]. With the development of the "precision medicine" surgical treatment approach [5], sentinel lymph node biopsy (SLNB), first introduced for breast cancer surgery by Krag et al. [6,7], has gradually replaced axillary surgery for early breast cancer treatment. It is an accurate method for assessing axillary lymph node status that could avoid severe complications and improve postoperative quality of life. However, only about $35 \%$ of all breast cancer patients who undergo SLNB have SLN metastases [8]. A retrospective study also showed that about $40 \%-70 \%$ of patients who had a positive SLN and accepted routine ALND had no tumor metastasis in their non-SLNs [9]. Therefore, the necessity of ALND after SLNB is still debated.

Preoperative prediction of SLN and non-SLN status has gained growing attention among doctors and scientists. Identifying patients who may not need ALND after SLNB requires an accurate method to predict non-SLN metastasis. Van et al. adopted the Memorial Sloan Kettering Cancer Center (MSKCC) model to predict the likelihood of nonSLN metastasis. This model included nine clinical or pathological variables and had an area under the curve (AUC) of 0.77 in a subsequent prospective study on 373 patients [10]. Many clinical centers have verified this model, but most found AUCs between 0.58 and 0.72 due to differences between countries and populations [10-12]. Most of the current non-SLN metastasis prediction models are based on patients from Western countries $[13,14]$. However, the numerous Chinese breast cancer patients differ from Western patients in race, diet, culture, and medical standards. Therefore, developing models suitable for Chinese populations is a necessity.

\section{Materials and Methods}

2.1. Case Collection. From November 2009 to December 2018, female patients $(n=736)$ who received SLNB were diagnosed as having primary breast cancer by preoperative or intraoperative biopsy in the Breast Cancer Center, Affiliated Cancer Hospital of Shantou University Medical College (Shantou, Guangdong Province, China). We recruited 228 of them for a retrospective training group. These cases fulfilled the following criteria: (1) primary invasive breast cancer was detected by preoperative needle biopsy or intraoperative freezing pathology, (2) patients met the cT1-3N0M0 stage according to the eighth edition of the American Joint Committee on Cancer (AJCC) staging manual, (3) patients had not received prior neoadjuvant therapy, (4) patients had undergone SLNB and tumor metastasis was observed in the SLNs, including isolated tumor cells, tumor micrometastases, or macrometastases, (5) an experienced surgical team performed the SLNB, (6) patients accepted further ALND, and (7) patients had no prior history of cancer. We continued to recruit 60 patients who met the inclusion criteria in our hospital's breast center

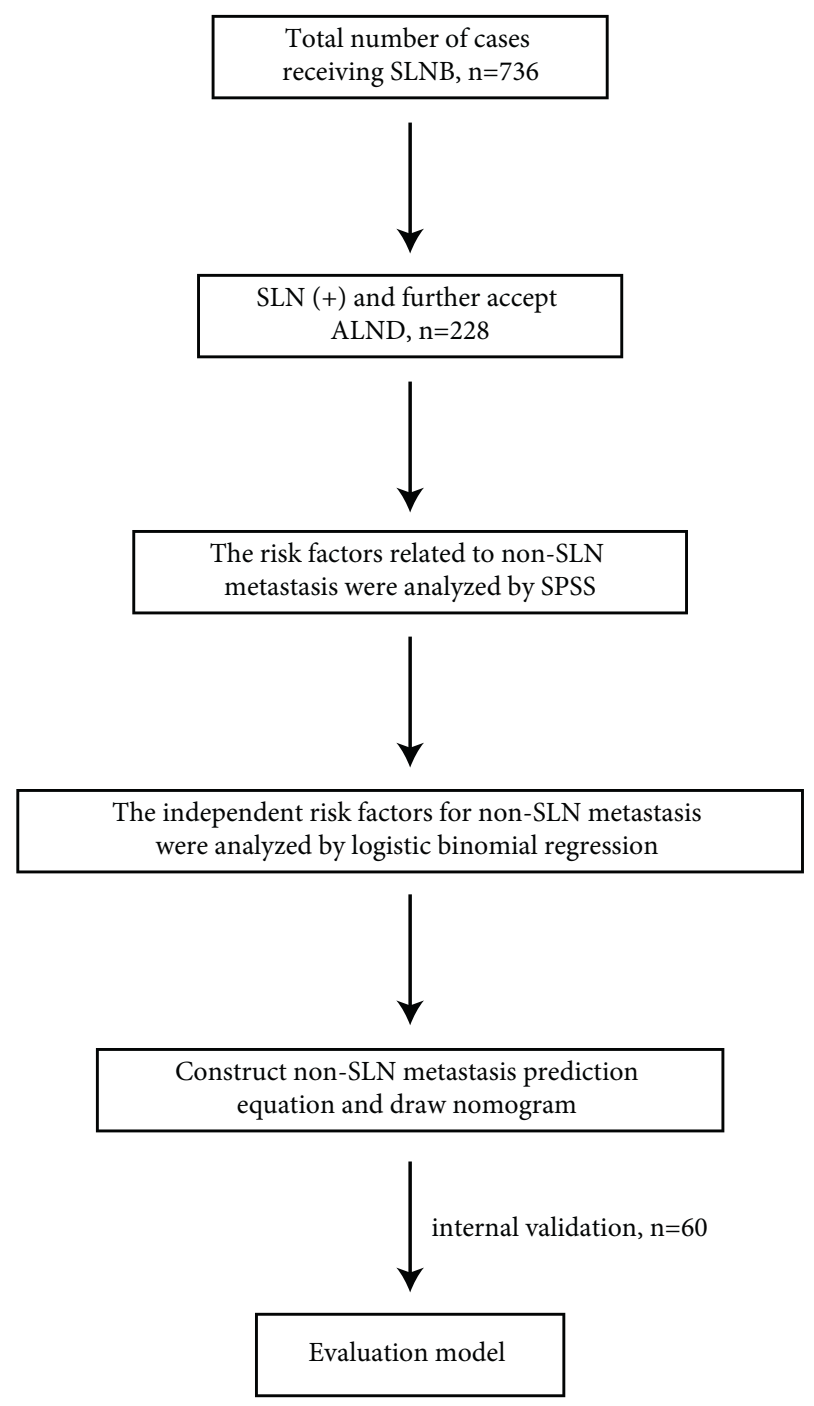

Figure 1: Project flow chart.

from January 2019 to July 2020 for preliminary verification of the model. The Ethics Committee of the Cancer Hospital of Shantou University Medical College approved this study (No. 2021102).

2.2. Surgery and Pathology. SLNB was performed using $2 \mathrm{ml}$ subcutaneously injected blue dye injection (Jichuan Pharmaceutical Group Co., Ltd., $10 \mathrm{mg} / \mathrm{mL}$, Jiangsu) and $2 \mathrm{ml}$ subcutaneously injected indocyanine green injection (Dandong Medical Innovation Pharmaceutical Co., Ltd., $12.5 \mathrm{mg} / \mathrm{ml}$, Liaoning). The staining tracer was injected into the areolar area, tumor surface, or subcutaneous tissue adjacent to the tumor, and SLNB was performed 5-10 minutes later. During SLNB, the main procedure consisted of looking for lymphatic vessels with blue staining or infrared irradiation in the axilla and then exploring the SLNs along the lymphatic vessels. We regarded stiff and swollen nodes near the blue-stained lymphatic vessels as the SLNs. A professional pathologist immediately examined all SLNs and communicated the result to the surgical team for a second 
TABLE 1: Comparison of clinicopathological characteristics between the model group and the validation group.

\begin{tabular}{|c|c|c|c|}
\hline Data & $\begin{array}{l}\text { Model } \\
\text { group }\end{array}$ & $\begin{array}{c}\text { Validation } \\
\text { group }\end{array}$ & $\begin{array}{c}P \\
\text { value }\end{array}$ \\
\hline \multicolumn{4}{|l|}{ Age } \\
\hline$\leq 35$ & 16 & 2 & \multirow{2}{*}{0.382} \\
\hline$>35$ & 212 & 58 & \\
\hline \multicolumn{4}{|l|}{ Menopausal status } \\
\hline Premenopausal & 116 & 32 & \multirow{2}{*}{0.735} \\
\hline Postmenopausal & 112 & 28 & \\
\hline \multicolumn{4}{|l|}{ Tumor location } \\
\hline Upper outer & 116 & 34 & \multirow{5}{*}{0.951} \\
\hline Lower outer & 32 & 7 & \\
\hline Lower inner & 13 & 3 & \\
\hline Upper inner & 43 & 11 & \\
\hline Central & 23 & 5 & \\
\hline \multicolumn{4}{|l|}{ Tumor size $(\mathrm{cm})$} \\
\hline Mean & 3.31 & 3.16 & \multirow{3}{*}{0.318} \\
\hline Median & 3 & 3 & \\
\hline $\mathrm{SD}$ & 1.29 & 0.95 & \\
\hline \multicolumn{4}{|l|}{ Tumor type } \\
\hline $\begin{array}{l}\text { Infiltrating ductal } \\
\text { carcinoma }\end{array}$ & 202 & 58 & \multirow{3}{*}{0.84} \\
\hline $\begin{array}{l}\text { Invasive lobular } \\
\text { carcinoma }\end{array}$ & 9 & 0 & \\
\hline Other carcinomas & 17 & 2 & \\
\hline \multicolumn{4}{|c|}{ Lymphovascular invasion } \\
\hline Yes & 31 & 2 & \multirow{2}{*}{0.023} \\
\hline No & 197 & 58 & \\
\hline \multicolumn{4}{|l|}{ Histological grade } \\
\hline G1 & 12 & 6 & \multirow{4}{*}{0.543} \\
\hline G2 & 69 & 19 & \\
\hline G3 & 136 & 33 & \\
\hline $\mathrm{Gx}$ & 11 & 2 & \\
\hline \multicolumn{4}{|l|}{ Multifocality } \\
\hline Yes & 18 & 1 & \multirow{2}{*}{0.139} \\
\hline No & 210 & 59 & \\
\hline \multicolumn{4}{|l|}{ Estrogen receptor } \\
\hline Negative & 59 & 15 & \multirow{2}{*}{0.890} \\
\hline Positive & 169 & 45 & \\
\hline Progesterone receptor & & & \\
\hline Negative & 87 & 18 & \\
\hline Positive & 141 & 42 & 0.243 \\
\hline HER2/neu receptor & & & \\
\hline Negative & 165 & 45 & \\
\hline Positive & 63 & 15 & 0.643 \\
\hline Ki-67 status & & & \\
\hline$\leq 14 \%$ & 33 & 12 & \\
\hline$>14 \%$ & 195 & 48 & 0.294 \\
\hline Molecular subtypes & & & \\
\hline Luminal A & 28 & 13 & \\
\hline Luminal B1 & 113 & 20 & \\
\hline Luminal B2 & 29 & 8 & 0.159 \\
\hline Her2-positive & 34 & 10 & \\
\hline Triple negativity & 24 & 9 & \\
\hline Number of SLN & & & \\
\hline Mean & 2.90 & 3.42 & \\
\hline Median & 3 & 3 & 0.016 \\
\hline $\mathrm{SD}$ & 1.48 & 1.44 & \\
\hline Number of metastatic & & & \\
\hline Mean & 1.43 & 1.87 & \\
\hline Median & 1 & 1 & 0.007 \\
\hline $\mathrm{SD}$ & 0.73 & 1.16 & \\
\hline
\end{tabular}

TABle 1: Continued.

\begin{tabular}{lccc}
\hline Data & $\begin{array}{c}\text { Model } \\
\text { group }\end{array}$ & $\begin{array}{c}\text { Validation } \\
\text { group }\end{array}$ & $\begin{array}{c}P \\
\text { value }\end{array}$ \\
\hline $\begin{array}{l}\text { Number of nonmetastatic } \\
\text { SLN }\end{array}$ & & & \\
$\quad$ Mean & 1.46 & 1.55 & \\
$\quad$ Median & 1 & 2 & 0.663 \\
$\quad$ SD & 1.35 & 1.32 & \\
Number of non-SLN & & & \\
$\quad$ Mean & 13.05 & 14.37 & \\
$\quad$ Median & 13 & 13 & 0.264 \\
SD & 5.31 & 5.63 & \\
\hline
\end{tabular}

judgment. In cases with tumor metastasis in the SLNs, we routinely dissected the level I or II axillary lymph nodes. If lymph nodes in level II displayed metastases, we also dissected axillary lymph nodes in level III [15]. After the operation, all specimens were paraffin-embedded for immunohistochemistry. We evaluated the pathological stage and molecular subtype of the tumor according to the St. Gallen International Expert Consensus on the Primary Therapy of Early Breast Cancer 2013 and College of American Pathologists clinical practice guideline [16, 17].

2.3. Data and Analysis. The flowchart of variable screening, nomogram model construction, and model evaluation is shown in Figure 1. We analyzed the data using SPSS 19.0 and performed a normality test before calculating the median, mean, and standard deviation. Enumeration data are expressed as frequencies or composition ratios. Ranked data are expressed as frequencies. We compared the data using a $\mathrm{t}$-test for two independent samples or nonparametric tests. Enumeration data were analyzed using the chi-square test or Fisher's exact test. Ranked data were analyzed using the ranksum test. The independent risk factors for non-SLN tumor metastasis were analyzed by logistic binomial regression. The first error level was set as $\alpha=0.05$ in all of the tests above, and $P<0.05$ was considered to indicate statistical significance.

\section{Results}

3.1. Patient Characteristics. In the training group, 228 female patients with early breast cancer had at least one positive SLN and underwent complete ALND. Among them, 112 were positive for non-SLN metastases after ALND, indicating that the non-SLN metastasis incidence was $49.1 \%$. The average tumor size was $3.31 \pm 1.29 \mathrm{~cm}$, and the average age was 51 years $(28-86$ years). We dissected 3,636 lymph nodes from patients who received ALND, with an average of $13.05 \pm 5.3$ per patient. The total dissected number of SLNs was 661, and the average number of total SLNs, positive SLNs, and negative SLNs was $2.90 \pm 1.48$, $1.43 \pm 0.73$, and $1.46 \pm 1.35$, respectively. The validation and training groups had similar data distributions (Table 1).

3.2. Correlation Analysis for Non-SLN Metastasis. According to the univariate analysis, the variables significantly associated with metastasis in the non-SLNs include age, clinical tumor size, multifocality, number of positive 
TABLE 2: Univariate analysis of non-SLN status in 228 SLN-positive patients with early breast cancer.

\begin{tabular}{|c|c|c|c|c|}
\hline Data & $\begin{array}{c}\text { Non-SLN metastasis; absent; } n=116 \\
(50.9 \%)\end{array}$ & $\begin{array}{c}\text { Non-SLN metastasis; present; } n=112 \\
(49.1 \%)\end{array}$ & $\begin{array}{c}\text { Total } \\
n=228\end{array}$ & $P$ value \\
\hline \multicolumn{5}{|c|}{ 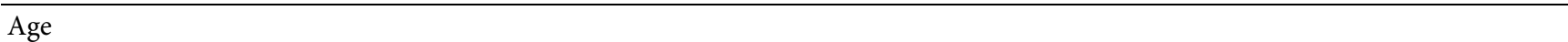 } \\
\hline$\leq 35$ & $12(10.3 \%)$ & $4(3.6 \%)$ & 16 & \multirow{2}{*}{0.045} \\
\hline$>35$ & $104(89.6 \%)$ & $108(96.4 \%)$ & 212 & \\
\hline \multicolumn{5}{|l|}{ Menopausal status } \\
\hline Premenopausal & $57(49.1 \%)$ & $59(52.7 \%)$ & 116 & \multirow{2}{*}{0.593} \\
\hline Postmenopausal & $59(50.9 \%)$ & $53(47.3 \%)$ & 112 & \\
\hline \multicolumn{5}{|l|}{ Tumor location } \\
\hline Upper outer & $57(49.6 \%)$ & $59(52.7 \%)$ & 114 & \multirow{5}{*}{0.828} \\
\hline Lower outer & $15(13.0 \%)$ & $17(15.2 \%)$ & 32 & \\
\hline Lower inner & $6(5.2 \%)$ & $7(6.2 \%)$ & 13 & \\
\hline Upper inner & $23(20.0 \%)$ & $20(17.9 \%)$ & 43 & \\
\hline Central & $14(12.2 \%)$ & $9(8.0 \%)$ & 23 & \\
\hline \multicolumn{5}{|l|}{ Tumor size $(\mathrm{cm})$} \\
\hline Mean & 3.03 & 3.59 & 3.31 & \multirow{3}{*}{0.828} \\
\hline Median & 3.00 & 3.40 & 3.00 & \\
\hline $\mathrm{SD}$ & 1.11 & 1.39 & 1.29 & \\
\hline \multicolumn{5}{|l|}{ Tumor type } \\
\hline $\begin{array}{l}\text { Infiltrating ductal } \\
\text { carcinoma }\end{array}$ & $105(90.5 \%)$ & $97(86.6 \%)$ & 202 & \multirow{3}{*}{0.214} \\
\hline Invasive lobular carcinoma & $2(1.7 \%)$ & $7(6.3 \%)$ & 9 & \\
\hline Other carcinomas & $9(7.8 \%)$ & $8(7.1 \%)$ & 17 & \\
\hline \multicolumn{5}{|l|}{ Lymphovascular invasion } \\
\hline Yes & $8(6.9 \%)$ & $23(20.5 \%)$ & 31 & \multirow{2}{*}{0.003} \\
\hline No & $108(93.1 \%)$ & $89(79.5 \%)$ & 197 & \\
\hline \multicolumn{5}{|l|}{ Histological grade } \\
\hline G1 & $7(6.0 \%)$ & $5(4.5 \%)$ & 12 & \multirow{4}{*}{0.841} \\
\hline $\mathrm{G} 2$ & $37(31.9 \%)$ & $32(28.5 \%)$ & 69 & \\
\hline G3 & $66(56.9 \%)$ & $70(62.5 \%)$ & 136 & \\
\hline $\mathrm{Gx}$ & $6(5.2 \%)$ & $5(4.5 \%)$ & 11 & \\
\hline \multicolumn{5}{|l|}{ Multifocality } \\
\hline Yes & $4(3.4 \%)$ & $14(12.5 \%)$ & 18 & \multirow{2}{*}{0.011} \\
\hline No & $112(96.6 \%)$ & $98(87.5 \%)$ & 210 & \\
\hline Estrogen receptor & & & & \\
\hline Negative & $34(29.3 \%)$ & $25(22.3 \%)$ & 59 & \\
\hline Positive & $82(70.7 \%)$ & $87(77.7 \%)$ & 169 & 0.228 \\
\hline Progesterone receptor & & & & \\
\hline Negative & $44(37.9 \%)$ & $43(38.4 \%)$ & 87 & \\
\hline Positive & $72(62.1 \%)$ & $69(61.6 \%)$ & 141 & 0.943 \\
\hline HER2/neu receptor & & & & \\
\hline Negative & $82(70.7 \%)$ & $83(74.1 \%)$ & 165 & \\
\hline Positive & $34(29.3 \%)$ & $29(25.9 \%)$ & 63 & 0.564 \\
\hline $\mathrm{Ki}-67$ status & & & & \\
\hline$\leq 14 \%$ & $19(16.4 \%)$ & $14(12.5 \%)$ & 33 & \\
\hline$>14 \%$ & $97(83.6 \%)$ & $98(87.5 \%)$ & 195 & 0.405 \\
\hline Molecular subtypes & & & & \\
\hline Luminal A & $16(13.8 \%)$ & $12(10.7 \%)$ & 28 & \\
\hline Luminal B1 & $51(44.0 \%)$ & $62(55.4 \%)$ & 113 & \\
\hline Luminal B2 & $17(14.7 \%)$ & $12(10.7 \%)$ & 29 & 0.415 \\
\hline Her2-positive & $17(14.7 \%)$ & $17(15.2 \%)$ & 34 & \\
\hline Triple negativity & $15(12.9 \%)$ & $9(8.0 \%)$ & 24 & \\
\hline Number of SLN & & & & \\
\hline Mean & 2.96 & 2.84 & 2.9 & \\
\hline Median & 3.0 & 3.0 & 3.0 & 0.558 \\
\hline SD & 1.53 & 1.43 & 1.48 & \\
\hline Number of metastatic SLN & & & & \\
\hline Mean & 1.31 & 1.56 & 1.43 & \\
\hline Median & 1 & 1 & 1 & 0.009 \\
\hline $\mathrm{SD}$ & 0.58 & 0.84 & 0.73 & \\
\hline Number of nonmetastatic SLN & & & & \\
\hline Mean & 1.65 & 1.28 & 1.46 & \\
\hline Median & 1 & 1 & 1 & 0.034 \\
\hline SD & 1.41 & 1.26 & 1.35 & \\
\hline
\end{tabular}


SLNs, number of negative SLNs, and lymphovascular invasion $(P<0.05)$ (Table 2). Multivariate analysis confirmed that clinical tumor size, multifocality, lymphovascular invasion, number of positive SLNs, and number of negative SLNs were independent predictors of non-SLN metastasis (Table 3).

\subsection{Establishment of a Predictive Model for Non-SLN} Metastasis. From the results of binary logistic analysis, we established the following predictive equation: $\operatorname{Logit}(P)=$ $\ln (P / 1-P)=0.267 \times a+1.443 \times b+1.078 \times c+0.471 \times d$ $-0.618 \times e-2.541$. During the calculation, we substituted the measured values of clinical tumor size, positive SLN number, and negative SLN number into the formula. We assigned a value of 0 or 1 for both multifocality and lymphovascular invasion, depending on the actual situation (Table 4). Using the binary logistic regression analysis results, using the " $R$ " language (version 3.5.3), we constructed a nomogram providing the probability of non-SLN metastasis (Figure 2).

3.4. Validation and Application of the Predictive Model. The AUC was 0.715 in the training group and 0.744 in the validation group, indicating consistent prediction performance in both groups (Figures 3(a) and 3(b)). Using the model in the clinic (to avoid ALND for low-risk SLN metastasis patients) requires setting a low-risk cutoff value in the model. When accepting a low-risk cutoff value of $\leq 14.2 \%$, about $2 \%$ of patients could be safely exempted from ALND, and the sensitivity was $100 \%$ in the training group. When accepting a low-risk cutoff value of $\leq 22.3 \%$, the sensitivity for both groups was above $90 \%$ and the falsenegative rate was below $10 \%$ (Tables 5 and 6 ). When accepting a low-risk cutoff value of $\leq 31.2 \%$ in the training group, the model correctly predicted that about $13 \%$ of patients had no non-SLN metastasis and the false-negative rate was below $10 \%$.

\section{Discussion}

With low trauma, high sensitivity, and high accuracy, SLNB has gradually replaced ALND as the preferred method of axillary treatment for most ALN-negative patients with early breast cancer [18]. Studies have pointed out that not all SLNpositive patients have non-SLN metastases. Only about $30 \%-50 \%$ of patients positive for SLN metastases have nonSLN metastases [19]. In our study, 112 patients (49.1\%) displayed non-SLN metastases, consistent with the literature [19]. Clinical trials such as IBCSG 23 [20] and Z0011 [21] have questioned the necessity of ALND for positive SLN patients and suggest that SLNB (alone or in combination with standard postoperative treatment) can achieve good local control without subsequent ALND for some SLNpositive low-risk populations.

The MSKCC nomogram is the most well-known multivariable model and has been used and verified in many hospitals, especially in Western countries. However, the AUC values obtained with the MSKCC nomogram fluctuate wildly depending on countries and populations. Wu et al. [13] used Chinese breast cancer patients to test the prognostic value of six standard models and obtained AUC values measured by the SNUH (Seoul National University Hospital), Louisville, MSKCC, Tenon, Stanford, and SCH (Shanghai Cancer Hospital) models of 0.706, 0.702, 0.677, $0.673,0.432$, and 0.674 , respectively. Thus, models have different predictive abilities, with AUC values ranging from 0.6 to 0.8 . Each model was constructed from clinical and pathological data for specific populations and therefore has the highest predictive value for that specific population but may not apply to other subjects. The SCH model is the first predictive non-SLN model in China and includes tumor size, number of negative SLNs, number of positive SLNs, vascular infiltration, and SLN tumor metastasis as variables. Although it achieved an AUC of 0.79 for its studied population, the results obtained for patients from other Chinese regions were not satisfactory $(A U C=0.674)[13,22]$. Our model achieved AUCs higher than 0.7 for both the training and validation groups. However, our validation group only contained 60 cases. Therefore, our model requires validation in other large independent populations before becoming feasible for clinical use. In the prediction model, each factor does not play a decisive role and each factor may affect lymph node metastasis. The clinical and pathological factors that may affect non-SLN metastasis are complex, with the most commonly associated risk factors being age, clinically positive lymph nodes, tumor size, tumor location, multifocality, pathologic type, neurovascular invasion, histological grade, number of positive and negative SLNs, positive SLN ratio, size of the SLN metastases detected, hormone receptor status, SLN micrometastases, and extracapsular invasion in the SLN [10, 23-26]. Among them, tumor size, neurovascular invasion, and positive SLN number have been common strong independent factors in multiple tests.

Our model involves only five independent factors, including the three essential factors mentioned above. It is simpler than previous models and avoids the interaction between too many variables, making it more widely applicable. As for the inclusion of multifocality, our model and the MSKCC, MDACC, and MOU models all indicate that multiple tumor foci impact non-SLN metastasis [27], although other investigators hold the opposite view [28]. This difference may be due to multifocality often being associated with large tumor volumes [29]. In our training group, the average tumor size of patients with multifocality was $3.64 \mathrm{~cm}$ (18/228). Moreover, a low occurrence rate of multiple foci may lead to distribution deviation. Differences in tumor cell aggressiveness, selection criteria, and sample size may also lead to different results.

Most models do not include the negative SLN number as a variable, the exceptions being the MSKCC and SCH models [22, 27]. Our model confirms that the negative SLN number significantly affects non-SLN metastasis. It is worth mentioning that the incidence of SLN metastasis is frequently accepted as an independent predictor, and both the Cambridge and Tenon models used this factor [26]. However, other studies tend to use the numbers for total SLNs, positive SLNs, and negative SLNs for analysis. The SLN 
TABLE 3: Multivariate analysis of non-SLN status in 228 SLN-positive patients with early breast cancer.

\begin{tabular}{|c|c|c|c|c|c|c|}
\hline Factors & Coefficient & S.E. & Wald & $P$ & OR & $95 \% \mathrm{CI}$ \\
\hline \multicolumn{7}{|l|}{ Age } \\
\hline$\leq 35$ & 1.000 & & & & 1.000 & \\
\hline$>35$ & 0.990 & 0.633 & 2.447 & 0.118 & 2.691 & $0.779-9.302$ \\
\hline Tumor size $(\mathrm{cm})$ & 0.267 & 0.123 & 4.734 & 0.030 & 1.307 & $1.027-1.663$ \\
\hline \multicolumn{7}{|l|}{ Multifocality } \\
\hline No & 1.000 & & & & 1.000 & \\
\hline Yes & 1.443 & 0.599 & 5.801 & 0.016 & 4.235 & $1.308-13.709$ \\
\hline \multicolumn{7}{|l|}{ Lymphovascular invasion } \\
\hline No & 1.000 & & & & 1.000 & \\
\hline Yes & 1.078 & 0.451 & 5.707 & 0.017 & 2.940 & $1.241-7.121$ \\
\hline Number of metastatic SLN & 0.471 & 0.221 & 4.552 & 0.033 & 1.602 & $1.039-2.469$ \\
\hline Number of nonmetastatic SLN & -0.618 & 0.242 & 6.519 & 0.011 & 0.539 & $0.336-0.866$ \\
\hline Constant & -2.541 & 0.802 & 10.030 & 0.002 & 0.079 & \\
\hline
\end{tabular}

TABLE 4: The assignment table of independent factors about non-SLN metastasis.

\begin{tabular}{lc}
\hline Factors & Assignment \\
\hline Tumor size $=a$ & $a=x_{1}, x_{1}(\mathrm{~cm})$ is the actual measured value in clinic \\
Multifocality $=b$ & Yes $=1 ;$ No $=0$ \\
Lymphovascular invasion $=c$ & Yes $=1 ;$ No $=0$ \\
Number of metastasis SLN $=d$ & $d=x_{2}, x_{2}$ is the number of metastasis SLN \\
Number of nonmetastasis SLN $=e$ & $e=x_{3}, x_{3}$ is the number of nonmetastasis SLN \\
\hline
\end{tabular}

metastasis rate actually combines the effect of positive SLNs and total SLNs, but the number of total SLNs does not necessarily affect the non-SLN state, as our results show. The SLN metastasis rate may also decrease the influence of the number of positive SLNs on the model. Therefore, we did not include it in our model.

Prior models rarely included age, menstrual state, and tumor location $[14,23,30]$. Our study yielded similar results. Breast cancer usually occurs in the breast's outer upper quadrant and rarely in the lower inner quadrant [31]. Although the outer upper quadrant is closer to the axilla, we did not find any correlation between tumor location and non-SLN metastasis. Metastasis development may depend more on the tumor's proliferative and invasive properties and the patient's internal environment. The classical MSKCC model includes the pathological subtype, which Mittendorf et al. [32] also regarded as an independent predictor. Some studies indicate that the pathology subtype does not affect non-SLN metastasis development [13, 25]. The failure to identify pathology subtype as an independent predictor in our study may be related to the different classifications of pathology subtypes. Insufficient sample size and uneven data distribution also make it challenging to reflect the pathology subtype's influence on non-SLN metastasis.

Histological grading, which involves assessing cancer cell division and differentiation, is an important indicator of cancer cell behavior. Although many investigators think that histological grading and lymph node metastasis may be related, the Cambridge model, which involved histological grading, had a multivariate analysis $P$ value of 0.050 [12]. Degnim et al. conducted a meta-analysis of 11 studies.
Among them, only one suggested that histological grading was correlated with non-SLN metastasis, while the others showed no correlation [33]. This study also supports the absence of correlation between histological grading and non-SLN metastasis. The higher the histological grading, the higher the degree of nuclear division, nuclear atypia, and vascularization. However, histological grading is often positively correlated with tumor size and vascular infiltration, so it is not necessarily an independent risk factor for non-SLN metastasis in statistical analyses. ER, PR, HER-2, and KI-67 are immunohistochemical indexes commonly associated with breast cancer and are closely related to patient treatment and prognosis. Most current models show no apparent correlation between KI-67 and the non-SLN status [13]. Whether the status of ER, PR, and HER-2 affects lymph node metastasis is still debated. ER was included in the nine variables in the MSKCC model, which analyzed the data of 11,946 patients and suggested that $\mathrm{PR}$ receptor status is related to axillary lymph node involvement [34]. Sandoughdaran et al. found HER-2 overexpression is related to non-SLN metastasis [35]. Mittendorf et al. and Fujii et al. pointed out that the ER/PR state and HER-2 expression are not significantly correlated with lymph node metastasis $[32,36]$. Few models include molecular typing in their variables [37].

Most current models have an AUC between 0.60 and 0.78. With 228 patients in the training group and 60 patients in the validation group, our model yielded AUC values higher than 0.7 for both groups, indicating good performance [38]. The ASCO guidelines published in 2005 pointed out that SLNB has an average false-negative rate of about $8.4 \%(0 \%-29 \%)$ [7]. Therefore, we reasoned that a false- 


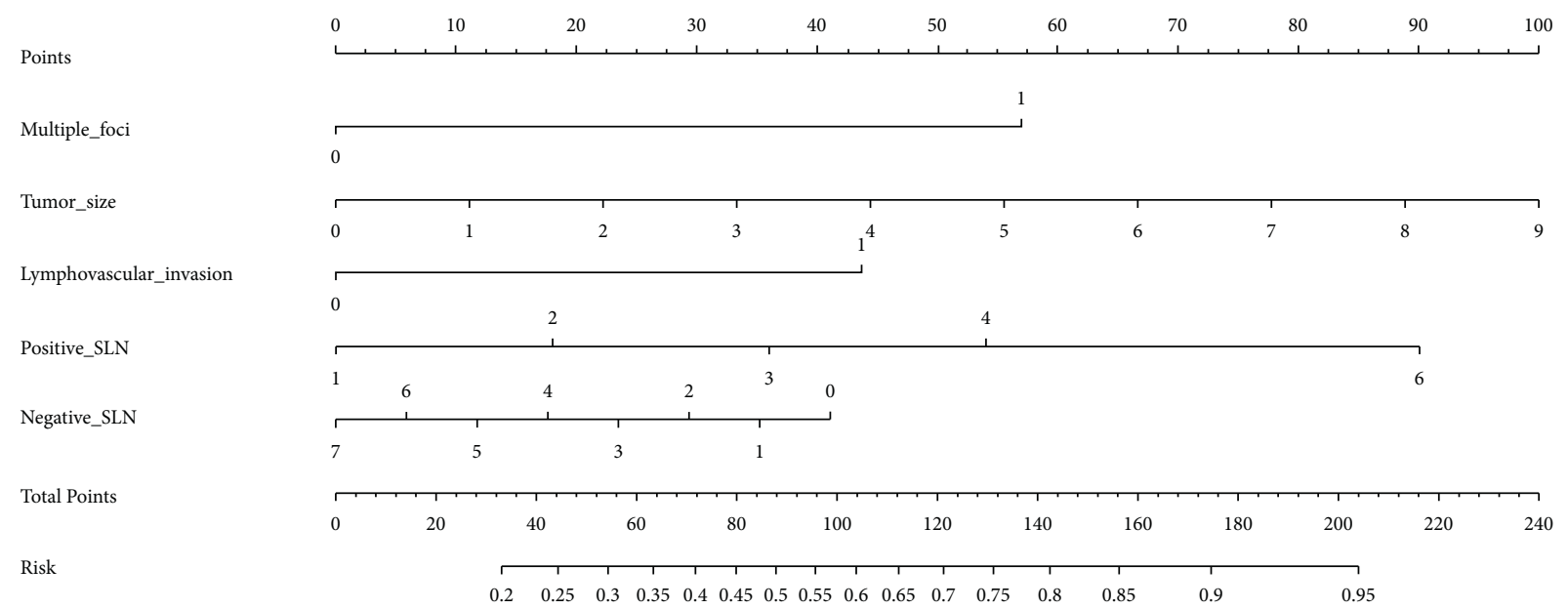

FIGURE 2: Nomogram for predicting the likelihood of non-sentinel lymph node (non-SLN) metastases in SLN-positive breast cancer patients. Substitute the variables according to the actual situation in rows 2 to 6 . The relative score for each variable is obtained by drawing a vertical line between each variable and the first row (Points). Then, the total points should be calculated and placed in row 7 . Finally, find the predicted $\mathrm{P}$, indicating the probability of non-SLN metastasis, in row 8.

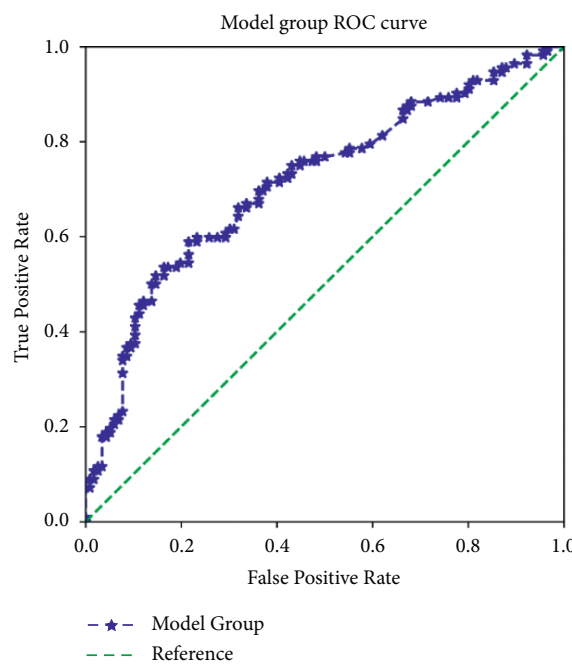

(a)

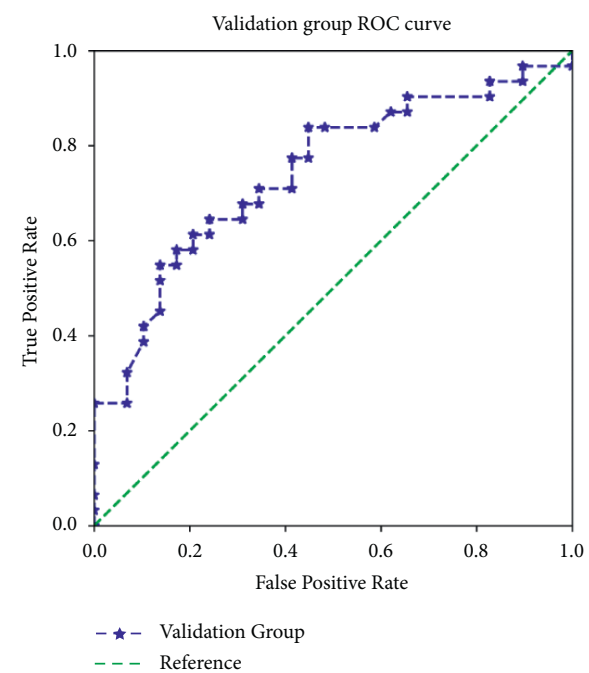

(b)

FIGURE 3: Receiver operating characteristic (ROC) curve, for the predictive equation, in the training group ((a) the area under the ROC curve was $0.715, n=228$ ) and validation group ((b) the area under the ROC curve was $0.744, n=60$ ).

TABle 5: Diagnostic evaluation table about risk interval from the predictive equation in the model group.

\begin{tabular}{lccccc}
\hline Cutoff values & Non-SLN metastasis; absent & Non-SLN metastasis; present & Sensitivity & Specificity & False-negative rate (\%) \\
\hline$\leq 14.2 \%$ & $4(2 \%)$ & 0 & $100.0 \%$ & $3.4 \%$ & $0.0 \%$ \\
$\leq 22.3 \%$ & $9(4 \%)$ & 2 & $98.2 \%$ & $6.8 \%$ & $1.8 \%$ \\
$\leq 28.6 \%$ & $17(7 \%)$ & 6 & $94.6 \%$ & $14.7 \%$ & $5.4 \%$ \\
$\leq 31.2 \%$ & $30(13 \%)$ & 11 & $90.2 \%$ & $22.4 \%$ & $3.8 \%$ \\
$\leq 35.7 \%$ & $39(17 \%)$ & 17 & $84.8 \%$ & $33.6 \%$ & $15.2 \%$ \\
& $n=228 ;$ among them, 112 patients were positive for non-SLNs with metastases \\
\hline
\end{tabular}

TABLe 6: Diagnostic evaluation table about risk interval from the predictive equation in the validation group.

\begin{tabular}{lccccc}
\hline Cutoff values & Non-SLN metastasis; absent & Non-SLN metastasis; present & Sensitivity & Specificity & False-negative rate (\%) \\
\hline$\leq 14.2 \%$ & $0(0 \%)$ & 0 & $100.0 \%$ & $0 \%$ & $0.0 \%$ \\
$\leq 22.3 \%$ & $10(17 \%)$ & 3 & $90.3 \%$ & $34.5 \%$ & $9.7 \%$ \\
$\leq 28.6 \%$ & $16(27 \%)$ & 6 & $80.6 \%$ & $55.2 \%$ & $19.4 \%$ \\
$\leq 31.2 \%$ & $17(28 \%)$ & 8 & $77.4 \%$ & $58.6 \%$ & $22.6 \%$ \\
$\leq 35.7 \%$ & $18(30 \%)$ & 9 & $58.6 \%$ & $29 \%$ \\
\multicolumn{5}{c}{$n$} \\
\hline
\end{tabular}


negative rate lower than $10 \%$ for axillary intervention would be acceptable to most physicians. Using the MSKCC and SCH models to explore low-risk interval [22], we found that, for risk values $\leq 10 \%$, their respective false-negative rates were $4.86 \%$ and $3.54 \%$. Furthermore, the corresponding proportion of patients was $8.10 \%$ and $13.6 \%$, respectively. For risk values $\leq 15 \%$, their respective false-negative rates were $13.54 \%$ and $8.20 \%$, and the corresponding proportion of patients was $16.2 \%$ and $30.0 \%$, respectively. In our model, for risk values $\leq 22.3 \%$, the false-negative rate in the training group was only $1.8 \%$, and the corresponding proportion of patients accounted for $4 \%$ of the total. For risk values $\leq 31.2 \%$, the model could accurately identify about $13 \%$ of patients without non-SLN metastasis, while the false-negative rate was also below $10 \%$.

Our model's low-risk interval value is higher than that of other models, but the proportion of patients avoiding ALND is lower. This phenomenon may be due to differences in the number and type of factors included in each model. In our model, the influence coefficients of multifocality and lymphovascular invasion are high, so the presence or absence of these two factors significantly impacts the prediction results. Furthermore, the training group contained only around 200 cases, making it hard to avoid the influence of risk interval division. However, our model can identify some non-SLNnegative patients with high accuracy as long as we strictly choose a low-risk cutoff value.

Axillary management of breast cancer has changed dramatically in the last decade. The IBCSG 23-01 trial in 2013 showed that if only SLN micrometastasis $(<2 \mathrm{~mm})$ occurs, omitting ALND does not negatively affect postoperative survival in early breast cancer patients [20]. In the EORTCAMAROS trial, SLN-positive early patients were divided into a radiotherapy group and an ALND group. The two groups had similar 5-year recurrence rates $(1.19 \% v s$. $0.43 \%)$, but the radiotherapy group patients had a significantly higher quality of life than the ALND group patients [39]. The ACOSOG Z0011 trial in 2016 showed that postoperative radiotherapy for patients who accepted breast-conserving surgery can replace ALND, even if SLNB detects one or two positive SLNs, with no significant difference in overall survival, disease-free survival, or local recurrence between the groups [21]. However, those trials had relatively strict inclusion conditions and their results do not apply to most patients. The development of additional models can help more patients appropriately avoid ALND. Our model, which incorporates five common variables, demonstrates good prognostic performance in assessing non-SLN metastatic risk in positive SLN patients. However, it requires more external validation in the future.

\section{Conclusion}

Our study developed a new prognostic model capable of predicting the nonsentinel lymph node (non-SLN) status of Chinese breast cancer patients. The equation for predicting non-SLN metastasis includes the following factors: tumor size, multifocality, lymphovascular invasion, number of SLNs with metastasis, and number of SLNs without metastasis. Our model demonstrates good prognostic performance in assessing the risk of non-SLN metastasis in patients with metastasis-positive SLNs.

\section{Data Availability}

All data of this study are available from the corresponding author upon request.

\section{Ethical Approval}

Approval of the study protocol was obtained from the Institutional Ethics Committee of Cancer Hospital of Shantou University Medical College (No. 2021102). This study was undertaken according to the ethical standards of the World Medical Association Declaration of Helsinki.

\section{Disclosure}

Lifang He and Peide Liang as co-first authors.

\section{Conflicts of Interest}

The authors declare no conflicts of interest.

\section{Authors' Contributions}

Lifang $\mathrm{He}$ and Peide Liang contributed equally to this work and are regarded as co-first authors. Yukun Cui and Wenhe Huang conceived and designed the research. Lifang He and Peide Liang fully participated in all aspects of the study, analyzed the data, and wrote the first draft of the paper. Huancheng Zeng and Guangsheng Huang analyzed the data and wrote part of the paper. Jundong $\mathrm{Wu}$ and Yiwen Zhang collected part of the data. All authors reviewed and approved the final manuscript.

\section{Acknowledgments}

This work was supported by the Shantou Science and Technology Program (No. 190819155262909), Guangdong Provincial Key Laboratory for Breast Cancer Diagnosis and Treatment (No. 2017B030314116), and Science and Technology Special Fund of Guangdong Province of China (No. 210728156901648).

\section{References}

[1] H. Sung, J. Ferlay, R. L. Siegel et al., "Global cancer statistics 2020: GLOBOCAN estimates of incidence and mortality worldwide for 36 cancers in 185 countries," CA: A Cancer Journal for Clinicians, vol. 73, no. 3, pp. 209-249, 2021.

[2] T.-A. Moo, R. Sanford, C. Dang, and M. Morrow, "Overview of breast cancer therapy," PET Clinics, vol. 13, no. 3, pp. 339-354, 2018.

[3] V. P. Chavda, Y. N. Ertas, V. Walhekar et al., "Advanced computational methodologies used in the discovery of new natural anticancer compounds," Frontiers in Pharmacology, vol. 12, Article ID 702611, 2021.

[4] D. N. Krag, S. J. Anderson, T. B. Julian et al., "Technical outcomes of sentinel-lymph-node resection and conventional axillary-lymph-node dissection in patients with clinically 
node-negative breast cancer: results from the NSABP B-32 randomised phase III trial," The Lancet Oncology, vol. 8, no. 10, pp. 881-888, 2007.

[5] Council NR. Toward Precision Medicine: Building a Knowledge Network for Biomedical Research and a New Taxonomy of Disease, Council NR, Washington (DC), US, 2011.

[6] S. P. Harlow, D. N. Krag, T. B. Julian et al., "Prerandomization Surgical Training for the National Surgical Adjuvant Breast and Bowel Project (NSABP) B-32 trial: a randomized phase III clinical trial to compare sentinel node resection to conventional axillary dissection in clinically node-negative breast cancer," Annals of Surgery, vol. 241, no. 1, pp. 48-54, 2005.

[7] G. H. Lyman, A. E. Giuliano, M. R. Somerfield et al., "American Society of Clinical Oncology guideline recommendations for sentinel lymph node biopsy in early-stage breast cancer," Journal of Clinical Oncology, vol. 23, no. 30, pp. 7703-7720, 2005.

[8] A. Stachs, K. Göde, S. Hartmann et al., "Accuracy of axillary ultrasound in preoperative nodal staging of breast cancer size of metastases as limiting factor," SpringerPlus, vol. 2, no. 1, p. 350, 2013.

[9] A. Maimaitiaili, D. Wu, Z. Liu, H. Liu, X. Muyiduli, and Z. Fan, "Analysis of factors related to non-sentinel lymph node metastasis in 296 sentinel lymph node-positive Chinese breast cancer patients," Cancer biology \& medicine, vol. 15, no. 3, pp. 282-289, 2018.

[10] K. J. Van Zee, D.-M. E. Manasseh, J. L. B. Bevilacqua et al., “A nomogram for predicting the likelihood of additional nodal metastases in breast cancer patients with a positive sentinel node biopsy," Annals of Surgical Oncology, vol. 10, no. 10, pp. 1140-1151, 2003.

[11] M. Klar, A. Jochmann, M. Foeldi et al., "The MSKCC nomogram for prediction the likelihood of non-sentinel node involvement in a German breast cancer population," Breast Cancer Research and Treatment, vol. 112, no. 3, pp. 523-531, 2008.

[12] A. Pal, E. Provenzano, S. W. Duffy, S. E. Pinder, and A. D. Purushotham, "A model for predicting non-sentinel lymph node metastatic disease when the sentinel lymph node is positive," British Journal of Surgery, vol. 95, no. 3, pp. 302-309, 2008.

[13] P. Wu, K. Zhao, Y. Liang, W. Ye, Z. Liu, and C. Liang, "Validation of breast cancer models for predicting the nonsentinel lymph node metastasis after a positive sentinel lymph node biopsy in a Chinese population," Technology in Cancer Research \& Treatment, vol. 17, Article ID 1533033818785032, 2018.

[14] L. Zhu, L. Jin, S. Li et al., "Which nomogram is best for predicting non-sentinel lymph node metastasis in breast cancer patients? A meta-analysis," Breast Cancer Research and Treatment, vol. 137, no. 3, pp. 783-795, 2013.

[15] W. J. Gradishar, B. O. Anderson, R. Balassanian et al., "NCCN guidelines insights: breast cancer, version 1.2017," Journal of the National Comprehensive Cancer Network, vol. 15, no. 4, pp. 433-451, 2017.

[16] A. C. Wolff, M. E. H. Hammond, D. G. Hicks et al., "Recommendations for human epidermal growth factor receptor 2 testing in breast cancer: American Society of Clinical Oncology/College of American Pathologists clinical practice guideline update," Journal of Clinical Oncology, vol. 31, no. 31, pp. 3997-4013, 2013.

[17] A. Goldhirsch, E. P. Winer, A. S. Coates et al., "Personalizing the treatment of women with early breast cancer: highlights of the St gallen international Expert Consensus on the primary therapy of early breast cancer 2013," Annals of Oncology, vol. 24, no. 9, pp. 2206-2223, 2013.

[18] U. Veronesi, G. Paganelli, G. Viale et al., "A randomized comparison of sentinel-node biopsy with routine axillary dissection in breast cancer," New England Journal of Medicine, vol. 349, no. 6, pp. 546-553, 2003.

[19] F. Biolchini, M. Vicentini, E. Di Felice et al., "Axillary nodal metastases in Italian early breast cancer patients with positive sentinel lymph node: can axillary node dissection be avoided by using predictive nomograms?" Tumori Journal, vol. 101, no. 3, pp. 298-305, 2015

[20] V. Galimberti, B. F. Cole, S. Zurrida et al., "Axillary dissection versus no axillary dissection in patients with sentinel-node micrometastases (IBCSG 23-01): a phase 3 randomised controlled trial," The Lancet Oncology, vol. 14, no. 4, pp. 297-305, 2013.

[21] A. E. Giuliano, K. Ballman, L. McCall et al., "Locoregional recurrence after sentinel lymph node dissection with or without axillary dissection in patients with sentinel lymph node metastases: long-term follow-up from the American College of surgeons oncology group (alliance) ACOSOG Z0011 randomized trial," Annals of Surgery, vol. 264, no. 3, pp. 413-420, 2016.

[22] J.-y. Chen, J.-j. Chen, J.-y. Xue et al., "Predicting non-sentinel lymph node metastasis in a Chinese breast cancer population with 1-2 positive sentinel nodes: development and assessment of a new predictive nomogram," World Journal of Surgery, vol. 39, no. 12, pp. 2919-2927, 2015.

[23] B. Yang, L. Yang, W. S. Zuo et al., "Predictors to assess nonsentinel lymph node status in breast cancer patients with only one sentinel lymph node metastasis," Chinese Medical Journal, vol. 126, no. 3, pp. 476-481, 2013.

[24] H. Jinno, M. Sakata, S. Asaga et al., "Predictors to assess nonsentinel lymph node status in breast cancer patients with sentinel lymph node metastasis," Breast Journal, vol. 14, no. 6, pp. 551-555, 2008.

[25] A. Reynders, O. Brouckaert, A. Smeets et al., "Prediction of non-sentinel lymph node involvement in breast cancer patients with a positive sentinel lymph node," The Breast, vol. 23, no. 4, pp. 453-459, 2014.

[26] A. Goyal, A. Douglas-Jones, R. G. Newcombe, R. E. Mansel, and A. T. Group, "Predictors of non-sentinel lymph node metastasis in breast cancer patients," European Journal of Cancer, vol. 40, no. 11, pp. 1731-1737, 2004.

[27] R. M. Nadeem, L. D. Gudur, and Z. A. Saidan, “An independent assessment of the 7 nomograms for predicting the probability of additional axillary nodal metastases after positive sentinel lymph node biopsy in a cohort of British patients with breast cancer," Clinical Breast Cancer, vol. 14, no. 4, pp. 272-279, 2014.

[28] R. F. Hwang, S. Krishnamurthy, K. K. Hunt et al., "Clinicopathologic factors predicting involvement of nonsentinel axillary nodes in women with breast cancer," Annals of Surgical Oncology, vol. 10, no. 3, pp. 248-254, 2003.

[29] T. Tot, "Axillary lymph node status in unifocal, multifocal, and diffuse breast carcinomas: differences are related to macrometastatic disease," Annals of Surgical Oncology, vol. 19, no. 11, pp. 3395-3401, 2012.

[30] Y. Zhou, X. Huang, F. Mao et al., "Predictors of nonsentinel lymph node metastasis in patients with breast cancer with metastasis in the sentinel node," Medicine, vol. 98, no. 1, Article ID e13916, 2019. 
[31] S. Wu, J. Zhou, Y. Ren et al., "Tumor location is a prognostic factor for survival of Chinese women with T1-2N0M0 breast cancer," International Journal of Surgery, vol. 12, no. 5, pp. 394-398, 2014.

[32] E. A. Mittendorf, K. K. Hunt, J. C. Boughey et al., "Incorporation of sentinel lymph node metastasis size into a nomogram predicting nonsentinel lymph node involvement in breast cancer patients with a positive sentinel lymph node," Annals of Surgery, vol. 255, no. 1, pp. 109-115, 2012.

[33] A. C. Degnim, K. A. Griffith, M. S. Sabel et al., "Clinicopathologic features of metastasis in nonsentinel lymph nodes of breast carcinoma patients," Cancer, vol. 98, no. 11, pp. 2307-2315, 2003.

[34] P. M. Ravdin, M. De Laurentiis, T. Vendely, and G. M. Clark, "Prediction of axillary lymph node status in breast cancer patients by use of prognostic indicators," JNCI Journal of the National Cancer Institute, vol. 86, no. 23, pp. 1771-1775, 1994.

[35] S. Sandoughdaran, M. Malekzadeh, and M. E. Mohammad Esmaeil, "Frequency and predictors of axillary lymph node metastases in Iranian women with early breast cancer," Asian Pacific Journal of Cancer Prevention: Asian Pacific Journal of Cancer Prevention, vol. 19, no. 6, pp. 1617-1620, 2018.

[36] T. Fujii, Y. Yanagita, T. Fujisawa, T. Hirakata, M. Iijima, and H. Kuwano, "Implication of extracapsular invasion of sentinel lymph nodes in breast cancer: prediction of nonsentinel lymph node metastasis," World Journal of Surgery, vol. 34, no. 3, pp. 544-548, 2010.

[37] N.-n. Wang, Z.-j. Yang, X. Wang et al., "A mathematical prediction model incorporating molecular subtype for risk of non-sentinel lymph node metastasis in sentinel lymph nodepositive breast cancer patients: a retrospective analysis and nomogram development," Breast Cancer, vol. 25, no. 6, pp. 629-638, 2018.

[38] M. Greiner, D. Pfeiffer, and R. D. Smith, "Principles and practical application of the receiver-operating characteristic analysis for diagnostic tests," Preventive Veterinary Medicine, vol. 45, no. 1-2, pp. 23-41, 2000.

[39] M. Donker, G. van Tienhoven, M. E. Straver et al., "Radiotherapy or surgery of the axilla after a positive sentinel node in breast cancer (EORTC 10981-22023 AMAROS): a randomised, multicentre, open-label, phase 3 non-inferiority trial," The Lancet Oncology, vol. 15, no. 12, pp. 1303-1310, 2014. 